\title{
Nontrivial Solutions of a Class of Kirchhoff Type Problems via Local Linking Method
}

\author{
Anmin Mao and Lin Zhang \\ School of Mathematical Sciences, Qufu Normal University, Qufu, Shandong 273165, China \\ Correspondence should be addressed to Anmin Mao; maoam@163.com
}

Received 29 August 2014; Accepted 23 November 2014; Published 10 December 2014

Academic Editor: Juan J. Nieto

Copyright ( 2014 A. Mao and L. Zhang. This is an open access article distributed under the Creative Commons Attribution License, which permits unrestricted use, distribution, and reproduction in any medium, provided the original work is properly cited.

The existence of nontrivial solutions of Kirchhoff type problems is proved by using the local linking method, and the new results do not require classical compactness conditions.

\section{Introduction}

This paper is concerned with the existence of nontrivial solutions of the following nonlocal Kirchhoff type problem:

$$
\begin{gathered}
-\left(a+b \int_{\Omega}|\nabla u|^{2}\right) \Delta u=f(x, u) \quad \text { in } \Omega ; \\
u=0 \quad \text { on } \partial \Omega,
\end{gathered}
$$

where $\Omega$ is a smooth bounded domain in $R^{n}(n=1,2), a, b>$ 0 , and $f(x, u): \bar{\Omega} \times R$ is a continuous real function. Similar nonlocal problems model several biological systems where $u$ describes a process which depends on the average of itself, for example, that of the population density; see [1].

Problem (1) is related to the stationary analogue of the equation

$$
u_{t t}-\left(a+b \int_{\Omega}|\nabla u|^{2}\right) \Delta u=g(x, u)
$$

proposed by Kirchhoff [2] as an extension of the classical D'Alembert wave equation for free vibrations of elastic strings. Kirchhoff's model takes into account the changes in length of the string produced by transverse vibrations. Some early classical studies of Kirchhoff equations were those of Bernstein [3] and Pohožaev [4]. However, (2) received great attention only after Lions [5] proposed an abstract framework for the problem. Some interesting results can be found in [6] and the references theorem. In recent years, Alves et al. [7] and $\mathrm{Ma}$ and Rivera [8] have obtained positive solutions of such problems by variational methods.

Later Perera and Zhang $[9,10]$ obtained nontrivial solutions of Kirchhoff type problems with asymptotically 4-linear term via Yang index. In [9] using the invariant sets of descent flow, Perera and Zhang got a positive, a negative, and a signchanging solution under the 4-sublinear case, asymptotically 4-linear case, and 4-superlinear case. In [10], the authors considered the 4-superlinear case:

$$
(A R): \exists v>4: v F(x, t) \leq t f(x, t), \quad|t| \text { large, }
$$

where $F(x, t)=\int_{0}^{t} f(x, s) d s$, which implies

$$
F(x, t) \geq C\left(|t|^{\nu}-1\right) .
$$

In [11], Mao and Zhang used minimax methods and invariant sets of decent flow to prove 4-superlinear Kirchhoff type problems without the PS condition and got multiple solutions. In [12], the paper studies the problem (1) by means of the Morse theory and local linking.

Motivated by [12-15] and by the new information concerning local linking, we study Kirchhoff type problems 4superlinear growth as $|u| \rightarrow \infty$. We make the following assumptions.

$\left(S_{1}\right)$ There exist $\delta>0, k \geq 1, c>0$, and $2<\alpha<4$ such that

$$
c u^{\alpha}+\frac{\lambda_{k}}{2} u^{2}<F(x, u)<\frac{\lambda_{k+1}}{2} u^{2} \text { for }|u|<\delta \text {. }
$$


$\left(S_{2}\right)\left(F(x, u) / u^{4}\right) \rightarrow+\infty$ as $|u| \rightarrow+\infty$ uniformly in $x \in$ $\Omega$.

$\left(S_{3}\right)$ There exist $r>4$ and $\mu>r-4$ such that

$$
\begin{gathered}
\limsup _{|u| \rightarrow \infty} \frac{F(x, u)}{|u|^{r}}<\infty \quad \text { uniformly for a.e. } x \in \Omega, \\
\liminf _{|u| \rightarrow \infty} \frac{(f(x, u) u-4 F(x, u))}{|u|^{\mu}}>0
\end{gathered}
$$

uniformly for a.e. $x \in \Omega$.

We are ready to state our main results.

Theorem 1. Assume that $\left(S_{1}\right)-\left(S_{3}\right)$ hold. Then problem (1) has at least one nontrivial solution.

Remark 2. Most of the results on the existence and multiplicity of solutions of (1) were obtained under the above superlinear condition $(A R)$ with or without the evenness assumption. Roughly speaking the role of $(A R)$ is to ensure the boundedness of all $(P S)$ (or $\left.\left(P S^{*}\right)\right)$ sequences for the corresponding functional. However, there are many functions which are superlinear but it is not necessary to satisfy $(A R)$ even if $1<\theta \leq 2$. For example,

$$
\begin{array}{r}
f(x, u)=\mu|u|^{\mu-2} u+(\mu-1)|u|^{\mu-3} u \sin ^{2} u+|u|^{\mu-1} \sin 2 u, \\
u \in R \backslash\{0\},
\end{array}
$$

where $\mu>2$. Then it is easy to check that $(A R)$ does not hold even for any $\theta>\mu-1>1$. On the other hand, in order to verify $(A R)$, it is usually an annoying task to compute the primitive function of $f$ and sometimes it is almost impossible. For example,

$$
f(x, u)=|u| u\left(1+e^{(1+|\sin u|)^{\alpha}}+|\cos u|^{\alpha}\right), \quad u \in R,
$$

where $\alpha>0$.

The aim of this paper is to deal with superlinear problems which do not satisfy $(A R) .\left(S_{2}\right)$ is weaker than $(A R)$ used in [10]. Without $(A R)$, it becomes more complicated. We do not know in our situations whether the Palais-Smale sequence is bounded. We replace $(P S)$ (or $\left.\left(P S^{*}\right)\right)$ sequences with weaker Cerami sequence or $(C)^{*}$-sequence (the definition given in the next section).

Remark 3. Compared with the method of invariant sets of descent flow and Morse theory, Yang index, our method is more simple and direct.

\section{Preliminaries}

Let $X$ be a real Banach space with a direct sum decomposition:

$$
X=X^{1} \oplus X^{2} .
$$

Consider two sequences of subspace:

$$
X_{0}^{1} \subset X_{1}^{1} \subset \cdots \subset X^{1}, \quad X_{0}^{2} \subset X_{1}^{2} \subset \cdots \subset X^{2}
$$

such that $X^{j}=\bigcup_{n \in N} X_{n}^{j}, j=1,2$, where $\operatorname{dim} X_{n}^{j}<\infty, j=$ $1,2, n \in N$.

For every multi-index $\alpha=\left(\alpha_{1}, \alpha_{2}\right) \in N \times N$, we denote by $X_{\alpha}$ the space

$$
X_{\alpha_{1}}^{1} \oplus X_{\alpha_{2}}^{2}
$$

We can know

$$
\alpha \leq \beta \Longleftrightarrow \alpha_{1} \leq \beta_{1}, \quad \alpha_{2} \leq \beta_{2} .
$$

A sequence $\left\{\alpha_{n}\right\} \subset N^{2}$ is admissible if, for every $\alpha \in N^{2}$, there is $m \in N$ such that

$$
n \geq m \Longrightarrow \alpha_{n} \geq \alpha
$$

For every $\varphi: X \rightarrow R$ we denote by $\varphi_{\alpha}$ the functional $\varphi$ restricted to $X_{\alpha}$.

Definition 4 (see [14]). Let $\varphi \in C^{1}(X, R)$. The function $\varphi$ satisfies $(C)^{*}$ condition if every sequence $\left\{u_{\alpha_{n}}\right\}$ such that $\alpha_{n}$ is admissible and

$$
u_{\alpha_{n}} \in X_{\alpha_{n}}, \quad \sup _{n} \varphi\left(u_{\alpha_{n}}\right)<\infty, \quad \varphi_{\alpha_{n}}^{\prime}\left(u_{\alpha_{n}}\right) \longrightarrow 0
$$

contains a subsequence which converges to a critical point of $\varphi$.

Definition 5 (see [15]). Let $X$ be Banach space with a direct sum decomposition $X=X^{1} \oplus X^{2}$. The functional $\varphi \in$ $C^{1}(X, R)$ has a local linking at 0 if, for some $r>0$,

$$
\begin{array}{lll}
\varphi(u) \geq 0 & u \in X^{1}, & \|u\| \leq r, \\
\varphi(u) \leq 0 & u \in X^{2}, & \|u\| \leq r .
\end{array}
$$

Lemma 6 (see [14]). Let $X$ be Banach space with a direct sum decomposition $X=X^{1} \oplus X^{2}$. Suppose that $\varphi \in C^{1}(X, R)$ satisfies the following assumptions.

$\left(I_{1}\right) \varphi$ has a local linking at 0 with respect to $\left(X^{1}, X^{2}\right)$.

$\left(I_{2}\right) \varphi$ satisfies $(C)^{*}$ condition.

$\left(I_{3}\right) \varphi$ maps bounded sets into bounded sets.

$\left(I_{4}\right)$ For every $m \in N, \varphi(u) \rightarrow-\infty$ as $u \in X_{m}^{1} \oplus X^{2}$ and $\|u\| \rightarrow \infty$.

Then $\varphi$ has at least one nontrivial critical point.

Next we let $H: H_{0}^{1}(\Omega)$ be the Sobolev space equipped with the inner product and the norm

$$
\langle u, v\rangle=\int_{\Omega} \nabla u \nabla v, \quad\|u\|=(u, u)^{1 / 2} .
$$

Denote by $0<\lambda_{1}<\lambda_{2}<\cdots$ the distinct Dirichlet eigenvalues of $-\Delta$ on $\Omega$, and denote by $\varphi_{1}, \varphi_{2}, \varphi_{3}, \ldots$ the eigenfunction corresponding to the eigenvalues; then

$$
\lambda_{1}(\Omega):=\inf _{u \in H,|u|_{2}=1}|\nabla u|_{2}^{2}
$$


is achieved by $\varphi_{1}>0$. Let

$$
E_{j}:=\bigoplus_{i \leq j} \operatorname{ker}\left(-\Delta-\lambda_{i}\right), \quad j \in \mathbb{N} .
$$

Recall that a function $u \in H$ is called a weak solution of (1) if

$$
\left(a+b\|u\|^{2}\right) \int_{\Omega} \nabla u \cdot \nabla v=\int_{\Omega} f(x, u) v, \quad \forall v \in H .
$$

Weak solutions are the critical points of $C^{1}$ functional:

$$
\varphi(u):=\frac{a}{2}\|u\|^{2}+\frac{b}{4}\|u\|^{4}-\int_{\Omega} F(x, u), \quad \forall u \in H .
$$

Then

$$
\begin{array}{r}
\varphi^{\prime}(u) v=\left(a+b\|u\|^{2}\right) \int_{\Omega} \nabla u \cdot \nabla v-\int_{\Omega} f(x, u) v, \\
\forall u, v \in H .
\end{array}
$$

\section{The Existence of the Solutions}

Lemma 7. Let $F$ satisfies $\left(S_{1}\right)$; the function $\varphi$ has a local linking with respect to $H=X^{1} \oplus X^{2}$, where $X^{1}=E_{k}, X^{2}=\left(X^{1}\right)^{\perp}$.

Proof. Let $X^{1}=E_{k}, X^{2}=\left(X^{1}\right)^{\perp}$. If $u \in X^{1}$, then $\|u\|^{2} \leq$ $\lambda_{k}|u|_{2}^{2}$; and if $u \in X^{2}$, then $\|u\|^{2} \geq \lambda_{k+1}|u|_{2}^{2}$. If $u \in X^{1}$, from $\left(S_{1}\right)$, for the given $\delta$, there exists a $\rho_{1}=\delta / c_{1}>0$ such that $\|u\| \leq \rho_{1} \Rightarrow|u| \leq c_{1}\|u\| \leq \delta$ and since $\operatorname{dim} X^{1}$ is finite $c|u|_{\alpha}^{\alpha} \geq c_{2}\|u\|^{\alpha}$; by $\left(S_{1}\right)$, we have

$$
\begin{aligned}
\varphi(u) & =\frac{a}{2}\|u\|^{2}+\frac{b}{4}\|u\|^{4}-\int_{\Omega} F(x, u) d x \\
& \leq \frac{a}{2}\|u\|^{2}+\frac{b}{4}\|u\|^{4}-\frac{a \lambda_{k}}{2}|u|_{2}^{2}-c|u|_{\alpha}^{\alpha} \\
& \leq \frac{b}{4}\|u\|^{4}-c_{2}\|u\|^{\alpha} .
\end{aligned}
$$

Noting that $2<\alpha<4$, we can choose $\rho_{2} \leq \rho_{1}$ small enough such that $\varphi(u)<0$ for $\|u\| \leq \rho_{2}$.

For $u \in X^{2}$, from $\left(S_{1}\right),\left(S_{3}\right)$, we have

$$
F(x, u) \leq \frac{a \lambda_{k+1}}{2} u^{2}+c_{3}|u|^{r}
$$

then

$$
\begin{aligned}
\varphi(u) & =\frac{a}{2}\|u\|^{2}+\frac{b}{4}\|u\|^{4}-\int_{\Omega} F(x, u) d x \\
& >\frac{a}{2}\|u\|^{2}+\frac{b}{4}\|u\|^{4}-\frac{a \lambda_{k+1}}{2}|u|_{2}^{2}-c_{3}|u|_{r}^{r} \\
& \geq \frac{b}{4}\|u\|^{4}-c_{4}\|u\|^{r} .
\end{aligned}
$$

For $r>4$, choosing $\rho_{3}$ small enough such that $(b / 4)\|u\|^{4}-$ $c_{4}\|u\|^{r}>0$ for $\|u\| \leq \rho_{3}$, we have $\varphi(u)>0$ for $u \in X^{2},\|u\| \leq$ $\rho_{3}$.

Choose $\rho=\min \left\{\rho_{2}, \rho_{3}\right\}$; it is very clear that $\varphi$ has a local linking at 0 with respect to $H=X^{1} \oplus X_{2}$.
Lemma 8. Suppose $\left(S_{3}\right)$ holds. Then function $\varphi$ satisfies condition $(C)^{*}$; that is, for every sequence $\left\{u_{\alpha_{n}}\right\} \subset X_{\alpha_{n}},\left\{u_{\alpha_{n}}\right\}$ has a convergent subsequence if $\left\{\varphi\left(u_{\alpha_{n}}\right)\right\}$ is bounded and $(1+$ $\left.\left\|u_{n}\right\|\right)\left\|\varphi_{\alpha_{n}}^{\prime}\left(u_{\alpha_{n}}\right)\right\| \rightarrow 0$ as $n \rightarrow \infty$, where $X_{\alpha_{n}}=X_{\alpha_{n}}^{1} \oplus X^{2}$, $X_{\alpha_{n}}^{1}=\overline{\operatorname{span}}\left\{e_{0}, e_{1}, \ldots, e_{n}\right\}$, and $\left(e_{i}\right)_{i \geq 0}$ is a Hilbert basis for $X^{1}$.

Proof. Suppose that $\left\{u_{\alpha_{n}}\right\} \subset X_{\alpha_{n}}, \varphi\left(u_{\alpha_{n}}\right)$ is bounded and $(1+$ $\left.\left\|u_{\alpha_{n}}\right\|\right)\left\|\varphi^{\prime}\left(u_{\alpha_{n}}\right)\right\| \rightarrow 0$ as $n \rightarrow \infty$; then there exists a constant $M>0$ such that

$$
\left|\varphi\left(u_{\alpha_{n}}\right)\right| \leq M, \quad\left(1+\left\|u_{\alpha_{n}}\right\|\right)\left\|\varphi^{\prime}\left(u_{\alpha_{n}}\right)\right\| \leq M
$$

for every $n \in N$. On the one hand, by (6), there is constant $c_{5}>0$ such that

$$
\lim _{\left|u_{0}\right| \rightarrow \infty} \sup _{|u|>u_{0}} \frac{F(x, u)}{|u|^{r}}<c_{5}
$$

then there exists $\delta_{1}>0, u_{0}>\delta_{1}$, such that

$$
\sup _{|u|>u_{0}} \frac{F(x, u)}{|u|^{r}}<c_{5}, \quad \frac{F(x, u)}{|u|^{r}}<c_{5} \quad \text { when }|u| \geq \delta_{1} .
$$

Hence

$$
F(x, u) \leq c_{5}|u|^{r} \quad \forall|u| \geq \delta_{1} \text {, a.e. } x \in \Omega \text {. }
$$

We know

$$
|F(x, u)| \leq M_{1} \quad \forall|u| \leq \delta_{1} \text {, a.e. } x \in \Omega \text {. }
$$

So for all $u \in R^{n}(n=1,2)$ and a.e. $x \in \Omega$, we obtain

$$
F(x, u) \leq c_{5}|u|^{r}+M_{1} .
$$

It follows from (26) and (31)

$$
\begin{aligned}
\frac{b}{4}\left\|u_{\alpha_{n}}\right\|^{4} & =\varphi\left(u_{\alpha_{n}}\right)+\int_{\Omega} F\left(x, u_{\alpha_{n}}\right) d x-\frac{a}{2}\left\|u_{\alpha_{n}}\right\|^{2} \\
& \leq \varphi\left(u_{\alpha_{n}}\right)+\int_{\Omega} F\left(x, u_{\alpha_{n}}\right) d x \\
& <M+M_{1}|\Omega|+c_{5} \int_{\Omega}\left|u_{\alpha_{n}}\right|^{r} d x .
\end{aligned}
$$

On the other hand, by (7),

$$
\lim _{\left|u_{0}\right| \rightarrow \infty} \inf _{|u|>u_{0}} \frac{f(x, u) u-4 F(x, u)}{|u|^{\mu}}>0 ;
$$

then there are constants $c_{6}>0$ and $\delta_{2}>0, u_{0}>\delta_{2}$, such that

$$
\begin{array}{r}
\inf _{|u|>u_{0}} \frac{f(x, u) u-4 F(x, u)}{|u|^{\mu}}>c_{6}, \\
\frac{f(x, u) u-4 F(x, u)}{|u|^{\mu}}>c_{6}
\end{array}
$$


Hence

$f(x, u) u-4 F(x, u) \geq c_{6}|u|^{\mu}>0 \quad \forall|u| \geq \delta_{2}$, a.e. $x \in \Omega$.

By $F \in C^{1}$, one has

$$
|f(x, u) u-4 F(x, u)| \leq M_{2}
$$

for every $|u| \leq \delta_{2}$, a.e. $x \in \Omega$.

Hence we obtain that

$$
f(x, u) u-4 F(x, u) \geq c_{6}|u|^{\mu}-M_{2}-c_{6} \delta_{2}^{\mu}
$$

for all $u \in R^{n}$ and a.e. $x \in \Omega$. Then we have

$$
\begin{aligned}
5 M \geq & 4 \varphi\left(u_{\alpha_{n}}\right)-\left(\varphi^{\prime}\left(u_{\alpha_{n}}\right), u_{\alpha_{n}}\right) \\
= & 2 a\left\|u_{\alpha_{n}}\right\|^{2}+b\left\|u_{\alpha_{n}}\right\|^{4} \\
& -4 \int_{\Omega} F\left(x, u_{\alpha_{n}}\right)-a\left\|u_{\alpha_{n}}\right\|^{2}-b\left\|u_{\alpha_{n}}\right\|^{4} \\
& +\int_{\Omega} f\left(x, u_{\alpha_{n}}\right) u_{\alpha_{n}} \\
= & \int_{\Omega} f\left(x, u_{\alpha_{n}}\right) u_{\alpha_{n}}-4 F\left(x, u_{\alpha_{n}}\right) d x+a\left\|u_{\alpha_{n}}\right\|^{2} \\
\geq & c_{6} \int_{\Omega}\left|u_{\alpha_{n}}\right|^{\mu} d x-M_{2}|\Omega|+a\left\|u_{\alpha_{n}}\right\|^{2}-c_{6} \delta_{2}^{\mu} .
\end{aligned}
$$

So $\int_{\Omega}\left|u_{\alpha_{n}}\right|^{\mu} d x$ is bounded. If $\mu \geq r$, by (32) and Hölder inequality,

$$
\int_{\Omega}\left|u_{\alpha_{n}}\right|^{r} d x \leq|\Omega|^{(\mu-r) / \mu}\left(\int_{\Omega}\left|u_{\alpha_{n}}\right|^{\mu} d x\right)^{r / \mu} .
$$

Thus we get that $\left\{\left\|u_{\alpha_{n}}\right\|\right\}$ is bounded. If $\mu \leq r$, we obtain

$$
\begin{aligned}
\int_{\Omega}\left|u_{\alpha_{n}}\right|^{r} d x & =\int_{\Omega}\left|u_{\alpha_{n}}\right|^{r-\mu} \cdot\left|u_{\alpha_{n}}\right|^{\mu} \\
& \leq \|\left. u_{\alpha_{n}}\right|_{\infty} ^{r-\mu} \int_{\Omega}\left|u_{\alpha_{n}}\right|^{\mu} d x \\
& \leq c_{7}\left\|u_{\alpha_{n}}\right\|^{r-\mu} \int_{\Omega}\left|u_{\alpha_{n}}\right|^{\mu} d x .
\end{aligned}
$$

Then by (32) and $r-\mu<4$, we know that $\left\{\left\|u_{\alpha_{n}}\right\|\right\}$ is bounded too. Hence $\left\{\left\|u_{\alpha_{n}}\right\|\right\}$ is bounded. Since $\left\{\left\|u_{\alpha_{n}}\right\|\right\}$ is bounded, for a subsequence, $\left\{u_{\alpha_{n}}\right\}$ converges to some $u_{\alpha}$ weakly in $H$ and strongly in $L^{s}(\Omega), 1 \leq s<+\infty$, and a.e. in $\Omega$ :

$$
\begin{aligned}
\varphi^{\prime}\left(u_{\alpha_{n}}\right) & \left(u_{\alpha}-u_{\alpha_{n}}\right) \\
= & \left(a+b\left\|u_{\alpha_{n}}\right\|^{2}\right) \int_{\Omega} \nabla u_{\alpha_{n}} \cdot \nabla\left(u_{\alpha}-u_{\alpha_{n}}\right) \\
& -\int_{\Omega} f\left(x, u_{\alpha_{n}}\right)\left(u_{\alpha}-u_{n}\right)
\end{aligned}
$$

Noting that $p \in(1,+\infty), q=p /(p-1)$,

$$
\begin{aligned}
\int_{\Omega}\left|f\left(x, u_{\alpha_{n}}\right)\left(u_{\alpha}-u_{\alpha_{n}}\right)\right| \leq & \left(\int_{\Omega}\left|f\left(x, u_{\alpha_{n}}\right)\right|^{q}\right)^{1 / q} \\
& \times\left(\int_{\Omega}\left|u_{\alpha}-u_{\alpha_{n}}\right|^{p}\right)^{1 / p} \\
\leq & \mathcal{c}_{7} \cdot\left|u_{\alpha}-u_{\alpha_{n}}\right|_{p} \longrightarrow 0
\end{aligned}
$$

as $n \rightarrow+\infty$. In addition,

$$
\begin{aligned}
\left(a+b\left\|u_{\alpha_{n}}\right\|^{2}\right) \int_{\Omega} \nabla u_{\alpha_{n}} \cdot \nabla\left(u_{\alpha}-u_{n}\right) \\
=\left(a+b\left\|u_{\alpha_{n}}\right\|^{2}\right)\left[\left(u_{\alpha_{n}}, u_{\alpha}\right)-\left(u_{\alpha_{n}}, u_{\alpha_{n}}\right)\right] \\
=\left(a+b\left\|u_{\alpha_{n}}\right\|^{2}\right) \\
\quad \times\left[\left(u_{\alpha_{n}}, u_{\alpha}\right)-\left(u_{\alpha}, u_{\alpha}\right)+\left(u_{\alpha}, u_{\alpha}\right)-\left(u_{\alpha_{n}}, u_{\alpha_{n}}\right)\right] .
\end{aligned}
$$

Since $\left\{u_{\alpha_{n}}\right\} \rightarrow u_{\alpha}$, then $\left(u_{\alpha_{n}}, u_{\alpha}\right)-\left(u_{\alpha}, u_{\alpha}\right)=\left(u_{\alpha_{n}}-u_{\alpha}, u_{\alpha}\right) \rightarrow$ 0 . The combination of (32)-(44) implies that

$$
\left(u_{\alpha_{n}}, u_{\alpha_{n}}\right) \longrightarrow\left(u_{\alpha}, u_{\alpha}\right), \quad j \longrightarrow+\infty
$$

Thus, $\left\{u_{\alpha_{n}}\right\} \rightarrow u_{\alpha}$ in $H$.

Lemma 9. $\varphi$ maps bounded sets into bounded sets.

Proof. From $\left(S_{3}\right)$, we know

$$
|F(x, u)| \leq c_{5}|u|^{r}+M_{1}
$$

so

$$
\begin{aligned}
\varphi(u) & =\frac{a}{2}\|u\|^{2}+\frac{b}{4}\|u\|^{4}-\int_{\Omega} F(x, u) d x \\
& \leq \frac{a}{2}\|u\|^{2}+\frac{b}{4}\|u\|^{4}+\int_{\Omega}\left(M_{1}+c_{5}|u|^{r}\right) \\
& \leq \frac{a}{2}\|u\|^{2}+\frac{b}{2}\|u\|^{4}+M_{1}|\Omega|+c_{8}\|u\|^{r} .
\end{aligned}
$$

Hence $\varphi$ maps bounded sets into bounded sets.

Lemma 10. For every $m \in N, \varphi(u) \rightarrow-\infty,\|u\| \rightarrow \infty$, $u \in X_{m}^{1} \oplus X^{2}$.

Proof. By condition $\left(S_{2}\right)$ and (2), there exist enough constants $M_{3}>0, M_{4}>0$, for all $(x, u) \in \Omega \times R^{n}$; we have

$$
F(x, u) \geq M_{3}|u|^{4}-M_{4} .
$$


So, for all $u_{0} \in X_{m}^{1} \oplus X^{2},\left\|u_{0}\right\|=1$, one has

$$
\begin{aligned}
\varphi\left(s u_{0}\right) & =\frac{a}{2}\left\|s u_{0}\right\|^{2}+\frac{b}{4}\left\|s u_{0}\right\|^{4}-\int_{\Omega} F\left(x, s u_{0}\right) d x \\
& \leq \frac{a}{2} s^{2}+\frac{b}{4} s^{4}-M_{3} s^{4} \int_{\Omega}\left|u_{0}\right|^{4}+M_{4}|\Omega| \\
& \leq \frac{a}{2} s^{2}+\frac{b}{4} s^{4}-M_{3} c_{9} s^{4}+M_{4}|\Omega| \\
& \leq \frac{a}{2} s^{2}+s^{4}\left(\frac{b}{4}-M_{3} c_{9}\right)+M_{4}|\Omega| \\
& \longrightarrow-\infty
\end{aligned}
$$

Hence, for every $m \in N, \forall u \in X_{m}^{1} \oplus X^{2}$, we have

$$
\varphi(u) \longrightarrow-\infty \text { as }\|u\| \longrightarrow \infty .
$$

Then the conclusion is obtained.

Proof of Theorem 1. By Lemmas 7-10 and Lemma 6, we complete the proof.

\section{Conflict of Interests}

The authors declare that there is no conflict of interests regarding the publication of this paper.

\section{References}

[1] M. Chipot and B. Lovat, "Some remarks on nonlocal elliptic and parabolic problems," Nonlinear Analysis, vol. 30, no. 7, pp. 46194627, 1997.

[2] G. Kirchhoff, Mechanik, Teubner, Leipzig, Germany, 1883.

[3] S. Bernstein, "Sur une classe d'équations fonctionnelles aux dérivées partielles," Izvestiya Rossiiskoi Akademii Nauk: Seriya Matematicheskaya, vol. 4, pp. 17-26, 1940.

[4] S. I. Pohožaev, "A certain class of quasilinear hyperbolic equations," Matematicheskii Sbornik, vol. 96, pp. 152-168, 1975.

[5] J. L. Lions, "On some questions in boundary value problems of mathematical physics," in Contemporary Developments in Continuum Mechanics and Partial Differential equations (Proc. Internat. Sympos, Inst.Mat, Univ. Fed. Rio de Janeiro, 1977), vol. 30 of North-Holland Mathematics Studies, pp. 284-346, NorthHolland, Amsterdam, The Netherlands, 1978.

[6] G. M. Figueiredo, N. Ikoma, and J. R. S. Júnior, "Existence and concentration result for the Kirchhoff type equations with general nonlinearities," Archive for Rational Mechanics and Analysis, vol. 213, no. 3, pp. 931-979, 2014.

[7] C. O. Alves, F. J. Corrêa, and T. F. Ma, "Positive solutions for a quasilinear elliptic equation of Kirchhoff type," Computers \& Mathematics with Applications, vol. 49, no. 1, pp. 85-93, 2005.

[8] T. F. Ma and J. E. M. Rivera, "Positive solutions for a nonlinear nonlocal elliptic transmission problem," Applied Mathematics Letters, vol. 16, no. 2, pp. 243-248, 2003.

[9] K. Perera and Z. Zhang, "Nontrivial solutions of Kirchhoff-type problems via the Yang index," Journal of Differential Equations, vol. 221, no. 1, pp. 246-255, 2006.
[10] Z. Zhang and K. Perera, "Sign changing solutions of Kirchhoff type problems via invariant sets of descent flow," Journal of Mathematical Analysis and Applications, vol. 317, no. 2, pp. 456463, 2006.

[11] A. Mao and Z. Zhang, "Sign-changing and multiple solutions of Kirchhoff type problems without the P.S. condition," Nonlinear Analysis: Theory, Methods \& Applications, vol. 70, no. 3, pp. 1275-1287, 2009.

[12] Y. Yang and J. Zhang, "Nontrivial solutions of a class of nonlocal problems via local linking theory," Applied Mathematics Letters, vol. 23, no. 4, pp. 377-380, 2010.

[13] Z.-L. Tao, S. Yan, and S.-L. Wu, "Periodic solutions for a class of superquadratic Hamiltonian systems," Journal of Mathematical Analysis and Applications, vol. 331, no. 1, pp. 152-158, 2007.

[14] S. Luan and A. Mao, "Periodic solutions for a class of nonautonomous Hamiltonian systems," Nonlinear Analysis: Theory, Methods \& Applications, vol. 61, no. 8, pp. 1413-1426, 2005.

[15] S. J. Li and M. Willem, "Applications of local linking to critical point theory," Journal of Mathematical Analysis and Applications, vol. 189, no. 1, pp. 6-32, 1995. 


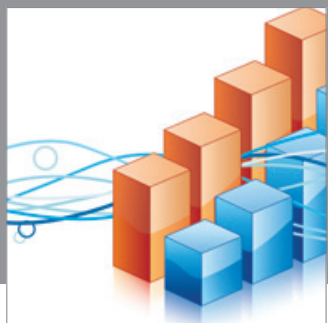

Advances in

Operations Research

mansans

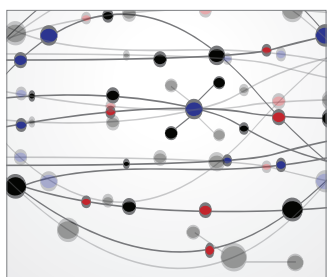

The Scientific World Journal
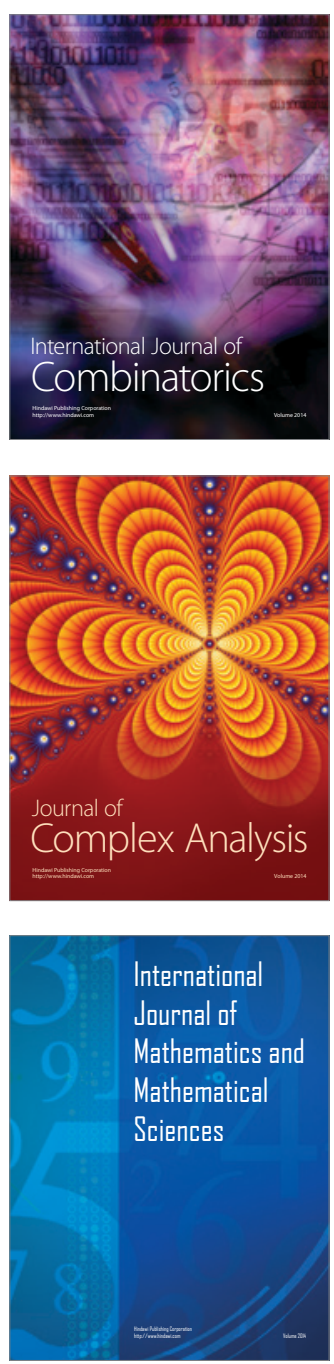
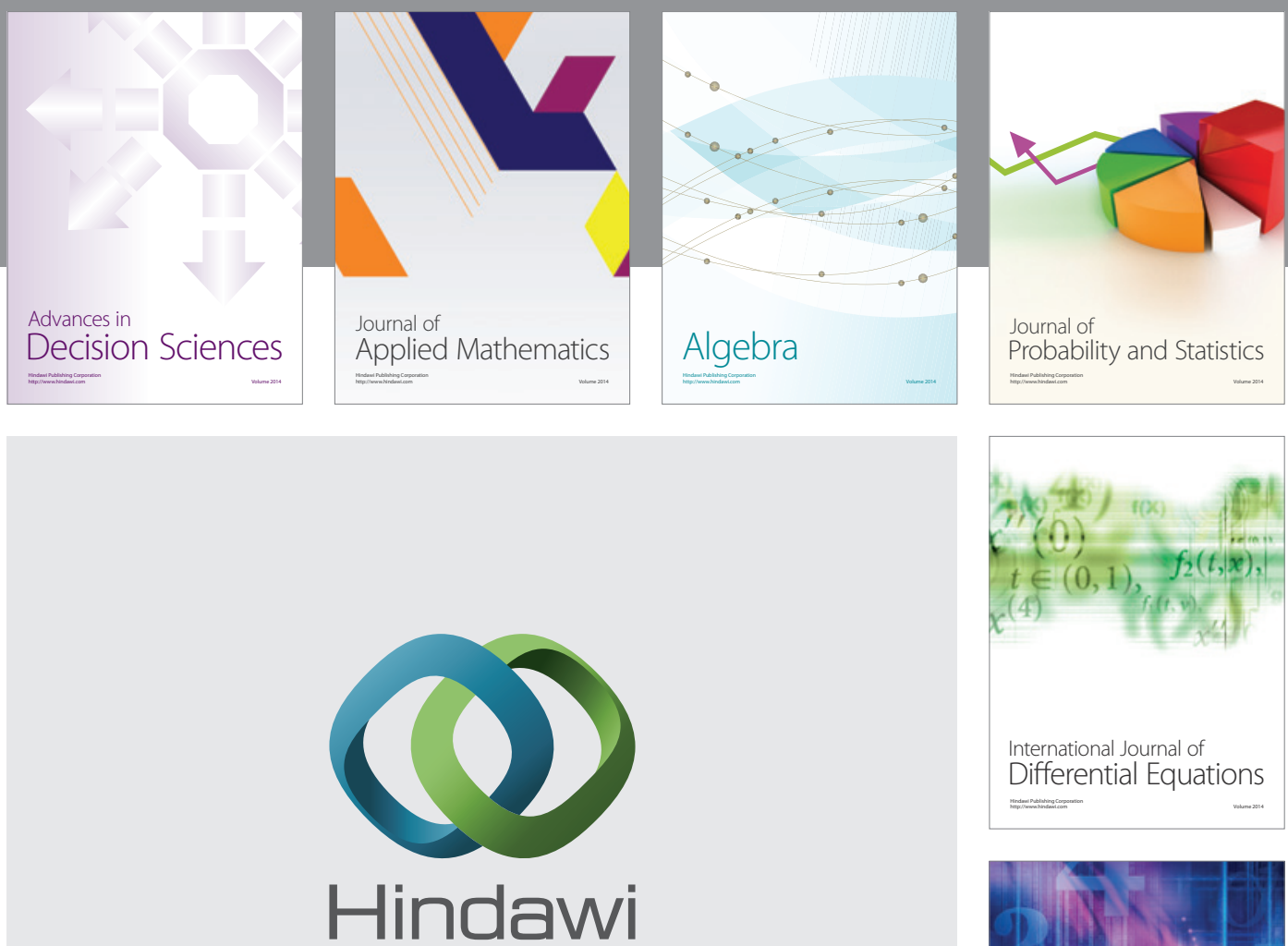

Submit your manuscripts at http://www.hindawi.com
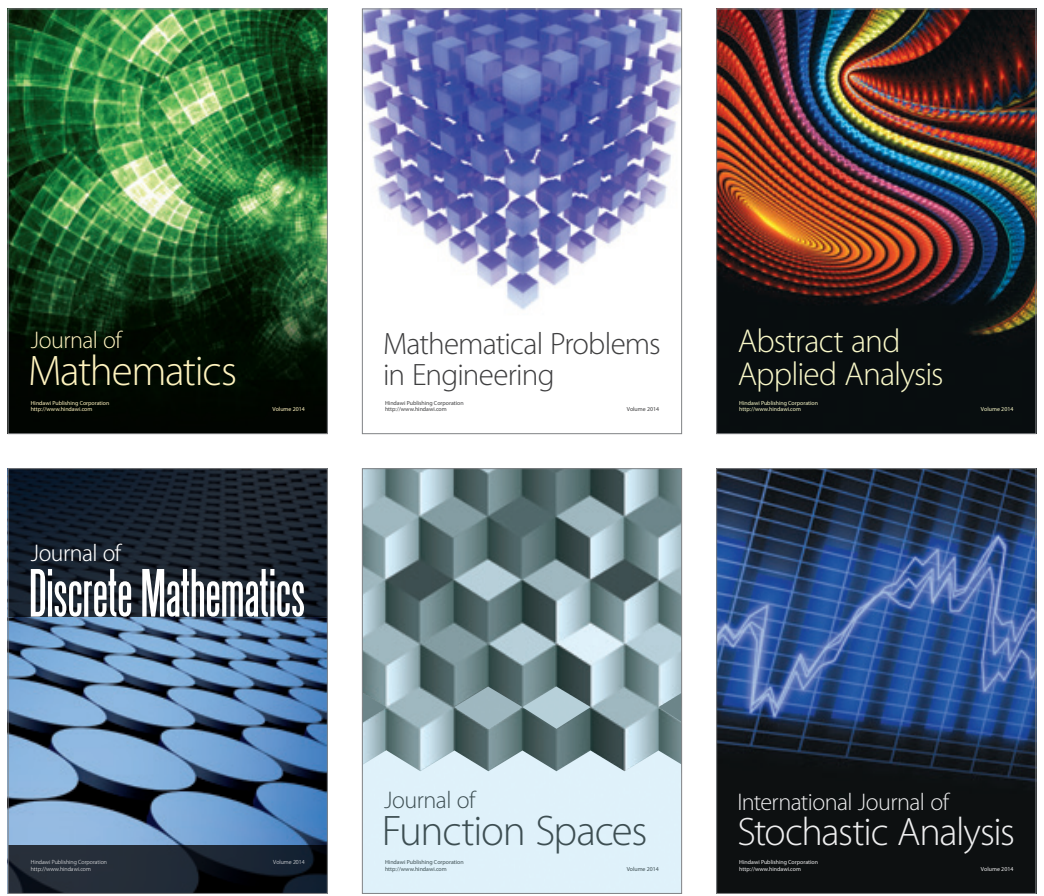

Journal of

Function Spaces

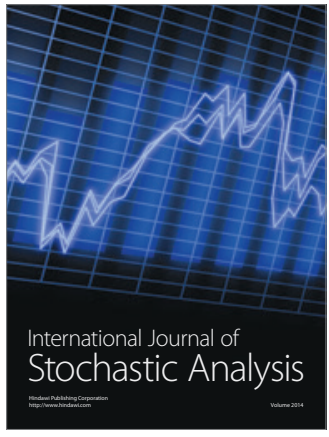

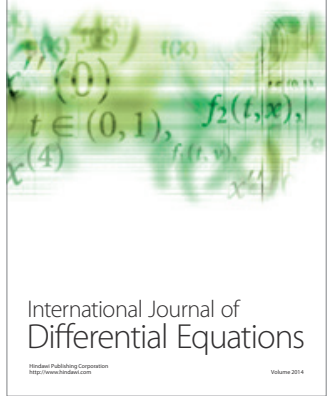
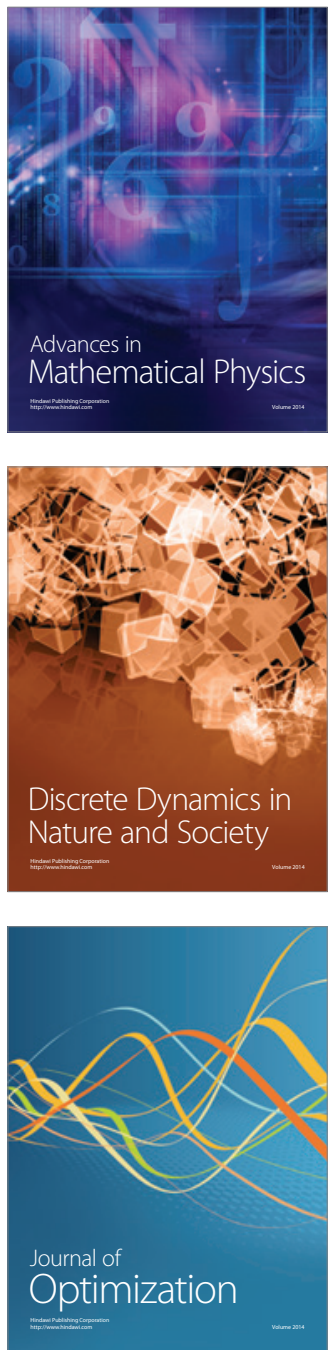